\title{
Vascular complications in Ehlers-Danlos syndrome type IV - case report
}

\author{
Katarzyna Grygiel, Maciej Jedrasik, Hamid Feiz Allah Poor, Slawomir Nazarewski, Jacek Szmidt \\ Department of General, Vascular and Transplant Surgery, Medical University of Warsaw, Poland
}

\begin{abstract}
Ehlers-Danlos syndrome is the most common inherited connective tissue disease and is a consequence of abnormal collagen synthesis. Type IV of this syndrome, called vascular, is considered to be the most severe. Patients with vascular type are prone to large arteries rupture and have higher likelihood of aneurysms development. We present a case of a patient with type IV Ehlers-Danlos syndrome, who suffered aortic rupture after surgical treatment of coeliac trunk compression syndrome. During the operation, the suturing the prosthesis to aorta was impossible due to the fragility of the aorta wall. Instead, a portion of a Dacron graft was used to envelop the aorta at the level of the rupture. Postoperative period was uneventful. The patient required delayed stent placement in common iliac artery due to its dissection. After 8 years of follow up patient remains in good condition.
\end{abstract}

Key words: Ehlers-Danlos syndrome, coeliac trunk compression syndrome abdominal aorta, aortic rupture, dissection

Acta Angiol 2015; 2I, 2: 6I-65

\section{Introduction}

Ehlers-Danlos syndrome is the most common inherited connective tissue disease, caused by abnormal collagen synthesis. Several types of the syndrome are recognized. The most severe form is type IV, called vascular, with an increased risk of rupture of large arteries and a high likelihood of aneurysm formation.

We present a case of a patient with type IV Ehlers-Danlos syndrome, in whom aortic rupture occurred after operation due to coeliac trunk compression syndrome.

\section{Case study}

A 49-year-old woman with type IV Ehlers-Danlos syndrome was admitted to the Department due to coeliac trunk compression syndrome. Symptoms of Ehlers-Danlos syndrome consisted of: thin and transparent skin, hypermobility of small joints and also positive family history. Four months before admission, she started to suffer from epigastric pain that appeared after meals. Computed tomography angiography (CTA) showed compression of the coeliac trunk by median arcuate ligament of the diaphragm and distension of infrarenal aorta to $30 \mathrm{~mm}$ (Fig. I).

The patient underwent elective surgery. Dissection of the median arcuate ligament eliminated compression. Significant fragility of the distended aorta was observed. During the first day after surgery, the patient suffered from severe abdominal pain, followed by cardiac arrest. After successful resuscitation, the patient was transferred to the intensive care unit. The abdominal ultrasound showed only a trace of fluid around the spleen and liver. For this reason, after stabilizing the patient's general condition, CTA of the abdomen was performed. It revealed a huge retroperitoneal haematoma (Fig. 2). Haematoma caused displacement of the right kidney laterally and anteriorly. 


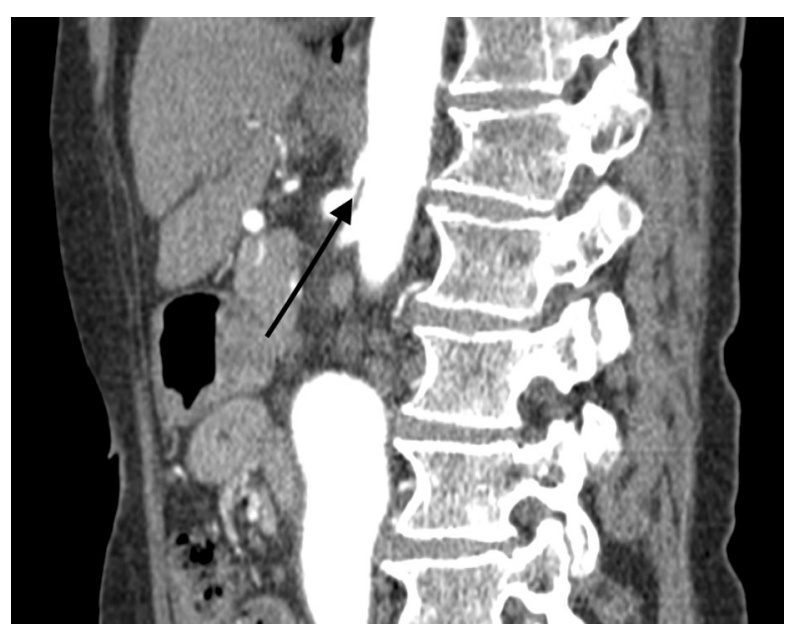

Figure I. Coeliac trunk compression (arrow)



Figure 2. Haematoma in retroperitoneal space

The patient was operated upon and rupture of the abdominal aortic aneurysm was found. In order to control bleeding, the aorta was closed below the diaphragm. The aorta was then dissected below the renal arteries and closed with a vascular clamp at the level of the left renal vein. At the level of the inferior mesenteric artery, there was a visible rupture of the aortic wall with a short dissection visible in its lumen. The fragility of the aorta wall prevented safe suturing of the prosthesis. For this reason, a portion of a Dacron ${ }^{\circledR}$ graft was used to envelop the aorta at the level of the rupture (Fig. 3). After releasing the vascular clamps, the flow through the aorta was normal with a well-palpable pulse over the femoral arteries. In the area of the aorta, haemostatic material was left, in order to facilitate improved haemostasis.

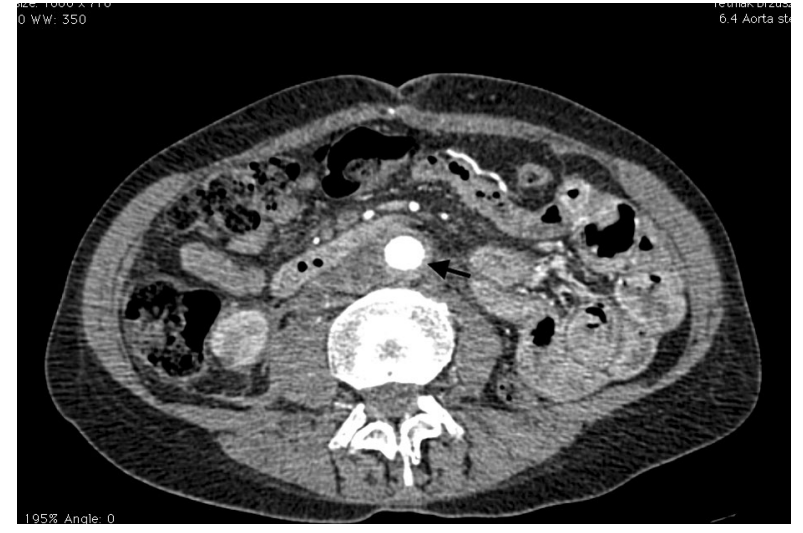

Figure 3. Aorta enveloped with portion of Dacron graft (arrow)

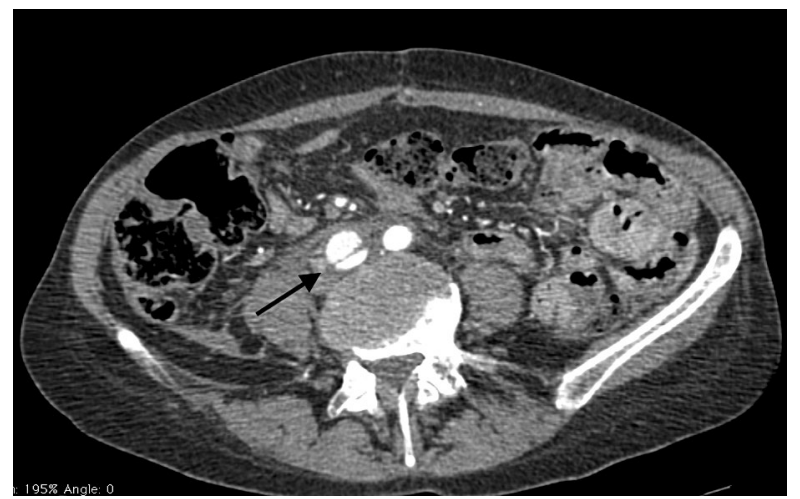

Figure 4. Dissection of right common iliac artery (arrow)

At the time of abdominal closure, there were no signs of bleeding from the aorta. In the control CTA, performed 3 days after the operation, normal blood flow through the aorta was shown, with no signs of extravasation. Retroperitoneal haematoma decreased significantly. The new finding was a dissection of the right common iliac artery (Fig. 4), starting at about $2 \mathrm{~cm}$ below the aortic bifurcation and ending at the level of its bifurcation, with no signs of leg ischaemia. Endovascular treatment of the dissection was planned, and the patient was discharged from the hospital 16 days after the initial surgery.

After 2 months, the patient was readmitted to the hospital for treatment of common iliac artery dissection. The control CTA scan was performed. It confirmed the presence of dissection of the iliac artery and also showed renal artery aneurysms on both sides (up to $6 \mathrm{~mm}$ in diameter) and in the splenic artery (up to $5 \mathrm{~mm}$ in diameter).

The dissection was treated with the use of a stent graft (GORE ${ }^{\circledR}$ VIABAHN ${ }^{\circledR}$ Endoprosthesis), $8 \times 100 \mathrm{~mm}$ 


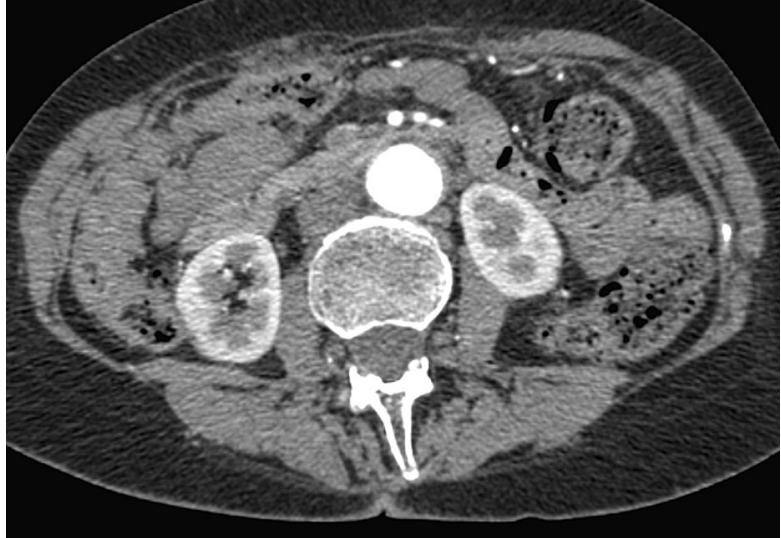

Figure 5. Abdominal aorta below renal arteries



Figure 6. Patent right common iliac and external iliac arteries

in size. During the endovascular procedure, it was necessary to cover the ostium of the right internal iliac artery. The control angiography revealed slight leakage into the false channel.

After procedure, the patient remained in good general condition; there were no features of limb ischaemia or claudication. The patient was discharged home with a recommendation for periodic visits to the outpatient clinic of the Department.

The next CTA scan was performed 2.5 months after the last procedure. The graft was patent, and there were no signs of leakage into the false channel. In control CTA performed 5 years after operation, maximal diameter of the aorta was $2.4 \times 2.7 \mathrm{~cm}$ (Fig. 5), stentgraft in right common iliac artery was patent (Fig. 6). Radiologist who assessed CTA saw no aneurysmal changes in splenic and renal arteries. Currently, after 8 years, the patient remains in good general health and Doppler ultrasound is performed every year to assess aorta and its branches.

\section{Discussion}

The first description of a patient with Ehlers-Danlos syndrome appeared in 1682. The Dutch surgeon Job van Meekeren described a patient with hyperextensible skin. In 190I, a Danish dermatologist named Ehlers and in 1908, a French physician named Danlos published additional cases of this disease. To date, II types of Ehlers-Danlos syndrome have been described [I, 2].

Ehlers-Danlos syndrome occurs at a frequency of $\mathrm{I}: 150,000$ births. Type IV is also known as vascular type. First described in 1936 by Sack [3], it represents 5-10\% of all cases of Ehlers-Danlos syndrome [4].

Clinical diagnosis of the vascular type of Ehlers-Danlos syndrome is based on four criteria.

Facial dysmorphy - acrogeria is the presence of prominent cheekbones and sunken cheeks. The eyes seem sunken or protruding because of a frequently occurring skin discoloration surrounding the orbit and telangiectasia on the eyelids. The nose and lips are narrow. There are cases of patients without facial acrogeria.

Symptoms associated with skin - the skin is abnormally thin and pale. It is also smooth, soft and velvety to the touch. The skin is translucent with visible veins. On the legs, the skin seems to be prematurely aged. In this type, there is no hyperextensibility of the skin. But the skin is fragile, though to a lesser extent than in the classical type of the syndrome. Thus, patients suffer from a long scarring process.

Haematomas and bruising are very common in patients with this type of Ehlers-Danlos syndrome.

\section{Vascular, gastrointestinal and gynaecological complications \\ Vascular complications}

The most common are arterial dissection and rupture of large and medium vessels [5], such as aortic arch branches, descending aorta and abdominal aorta, and rarely visceral, iliac, and femoral arteries [6, 7]. Most of the aneurysms are false. But true aneurysm also occurs [8]. Dissection of the vertebral arteries and the extracranial portions of carotid arteries is also characteristic of type IV Ehlers-Danlos syndrome [9]. The sudden rupture of large vessels and dissections are a frequent cause of death in these patients [10]. Sudden ruptures may be caused by minor trauma or effort [6].

Complications associated with the digestive tract Perforation in the large intestine [ I I, I 2], less frequently in the small intestine. Spontaneous rupture of the spleen and liver was also described [13]. 


\section{Gynaecological complications}

Pregnancy increases the risk of uterine rupture in women with type IV Ehlers-Danlos syndrome, especially during the last trimester [14].

Type IV Ehlers-Danlos syndrome is caused by heterozygous mutations in the COL3AI gene, which is inherited in an autosomal dominant fashion [15]. The COL3AI gene encodes a type III collagen. This type of collagen is a component of vessel walls and occurs in considerable amounts in the wall of the gastrointestinal tract.

A clinical diagnosis is based on the criteria developed by Villefranche [16]. Major criteria include fragility of the large vessels, gastrointestinal wall and uterus, and spontaneous rupture of these organs, thin and transparent skin, excessive bruising, and characteristic facial features. The smaller criteria are positive family history, acrogeria, hypermobility of small joints, and ruptures in the tendons and muscles.

The patient, whose case we present, fulfilled the criteria of type IV Ehlers-Danlos syndrome. The diagnosis was based, in addition to vascular changes, on other symptoms, such as thin and transparent skin, hypermobility of small joints, and a positive family history.

Rupture of the enlarged to $30 \mathrm{~mm}$ infrarenal portion of the abdominal aorta occurred during the first day after surgery due to coeliac trunk compression syndrome. The operation consisted of cutting the median arcuate ligament of the diaphragm and dissection of the fibres surrounding the coeliac trunk. Rupture of the aorta is difficult to connect to any dissection performed during operation on the coeliac trunk. On the other hand, it is known that even a small trauma to the vessels can cause its rupture [10].

Possibly dissection of the right common iliac artery played an important role in the rupture of the aorta and the creation of a huge retroperitoneal haematoma. This hypothesis is supported by the efficacy of the treatment of a ruptured aorta by wrapping it using piece of Dacron graft.

Most of the patients with vascular complications are operated on urgent basis [8]. As stated before, surgeon will encounter very fragile vessels thus atraumatic and gentle dissection is recommended. Most of the reports found in literature show either poor outcome from attempt to vascular reconstruction using grafts [10] or at least higher possibility of bleeding from anastomosis in early postoperative period and late graft related complications such as false aneurysm or ruptures at the level of anastomosis [8]. Authors advice special techniques to avoid tearing the arteries with vascular clamps and recommend avoiding the use of vein for reconstruction as it is also affected [17].
We think that the method used in presented case - wrapping the aorta with piece of Dacron ${ }^{\circledR}$ graft seems to be a good alternative to attempts of suturing the graft to the vessel in emergency conditions reducing this way surgical trauma and possible further vessel rupture. The idea of using a piece of material to secure aorta was shown to be efficient in treating acute ascending type A aorta dissection [18].

Endovascular techniques seem to be an interesting alternative for open repair and can be safely performed in patients with Ehlers-Danlos syndrome. Although an attention must be put on the access technique because of possible formation of false aneurysm, bleeding and dissection in place of puncture. In patients with vascular type of Ehlers-Danlos syndrome some authors recommend use of open access technique to avoid those complications [19]. In our patient we did not observe any setback after percutaneous puncture with $6 \mathrm{~F}$ sheaths and use of Angioseal ${ }^{\circledR}$.

\section{Conclusions}

Elective operations in patients with vascular Ehlers-Danlos syndrome, particularly type IV, are connected with a high risk of vascular complications during the postoperative period. Operations utilizing vascular grafts are difficult to perform due to the fragility of the vessels. Endovascular treatment appears to be a valuable alternative to classical methods.

Patients with Ehlers-Danlos syndrome require close observation during the postoperative period. According to the literature, clinical features of bleeding require more accurate diagnosis, not only of the vascular system, but also of the control of solid organs.

Periodic monitoring (clinical and radiological) and appropriate patient education, appears to be necessary to ensure effective long-term care of these patients.

\section{References}

I. Hakim JA, Sahota A (2006) Joint hypermobility and skin elasticity: the hereditary disorders of connective tissue. Clin Dermatol; 24: 52I-533.

2. Badauy CM, Gomes SS, Silho MS, Chies JAB (2007) Ehlers-Danlos Syndrome (EDS) type IV. Review of the literature. Clin Oral Invest; II: 183-187.

3. Sack G (1936) Status dysvascularis, ein Fall von besonderer Zerrei Blichkeit der Blutgafaße'. Dtsch Arch Klin Med; 178: 663-669.

4. Barabas AP (1972) Vascular complications in Ehlers-Danlos syndrome with special reference to the arterial type or Sack syndrome. J Cardiovasc Surg; 12: 160-167.

5. Bergqvist D (1996) Ehlers-Danlos type IV syndrome. A review from a vascular surgical point of view. Eur J Surg; 162: 163-170.

6. Cikrit DF, Miles JH, Silver D (1987) Spontaneous arterial perforation: the Ehlers-Danlos specter. J Vasc Surg; 5: 248-255. 
7. Habib K, Memon MA, Reid DA, Fairbrother BJ (200I) Spontaneous common iliac arteries rupture in Ehlers-Danlos syndrome type IV: report of two cases and review of the literature. Ann R Coll Surg Engl; 83: 96-104.

8. Oderich GS, Panneton JM, Bower TC et al (2005) The spectrum, management and clinical outcome of Ehlers-Danlos syndrome type IV: a 30-year experience. J Vasc Surg; 42: 98-106.

9. Schievink WI (2004) Cerebrovascular involvement in Ehlers-Danlos syndrome. Curr Treat Options Cardiovasc Med; 6: $231-236$.

10. Karkos CD, Prasad V, Mukhopadhyay U, Thomson GJ, Hearn AR (2000) Rupture of the abdominal aorta in patients with Ehlers-Danlos sydrome. Ann Vasc Surg; 14: 274-277.

II. Kinnane J, Priebe C, Caty M, Kuppermann N (1995) Perforation of the colon in an adolescent girl. Pediatr Emer Care; II: 230-232.

12. Berney T, La Scala G, Vettorel D et al (1994) Surgical pitfall in a patient with type IV Ehlers-Danlos syndrome and spontaneous colonic rupture. Report of a case. Dis Colon Rectum; 37: 1038-1042.
13. Harris SC, Slater DN, Austin CA (1985) Fatal splenic rupture in Ehlers-Danlos syndrome. Postgrad Med J; 61: 259-260.

14. Peaceman AM, Cruikshank DP (1987) Ehlers-Danlos syndrome and pregnancy: association of type IV disease with maternal death. Obstet Gynecol; 69: 428-43I.

15. Germain DP, Herrera-Guzman Y (2004) Vascular Ehlers-Danlos syndrome. Ann Genet; 47: I-9.

16. Beighton P, De Paepe A, Steinmann B, Tsipouras P, Wenstrup RJ (1998) Ehlers-Danlos syndromes: revised nosology, Villefranche, 1997. Am J Med Genet; 7: 31-37.

17. Brooke BS, Arnaoutakis GJ, Nazli A, McDonnell NB, Black JH III (2010) Contemporary management of vascular complications associated with Ehlers-Danlos syndrome. J Vasc Surg; 51: 131-139.

18. Demondion P, Ramadan R, Azmoun A et al (2014) Aortic wrapping for Stanford type A acute aortic dissection: short and midterm outcome. Ann Thorac Surg; 97: 1590-1596.

19. Lum YW, Brooke BS, Arnaoutakis GJ, Williams TK, Black JH III (2012) Endovascular procedures in patients with Ehlers-Danlos syndrome: a review of clinical outcomes and iatrogenic complications. Ann Vasc Surg; 26: 25-33. 